\title{
Intermittent hypoxic training: risks versus benefits
}

\author{
Helmut Hinghofer-Szalkay
}

Accepted: 22 October 2009 / Published online: 10 November 2009

(C) Springer-Verlag 2009

Large interindividual variations exist in human performance at high altitude. Some of these differences may be associated with certain genetic variants/genotype combinations (Gomez-Gallego et al. 2009). Studying microvascular, cellular and metabolic mechanisms of adaptation to high altitude helps to better understand individual physiological responses to hypoxia in high-performance athletes as well as in patients suffering from critical illness. Investigations such as the study presented by Sanchis-Gomar add valuable information on the underlying aspects of human physiology. There is an obvious effect on oxygen transport capacity, comparable in magnitude and quality to external supply with erythropoietin. The general question, of course, remains whether doping measures-even if as 'natural' as intermittent reduction of available oxygen — should be seen as a model for therapeutic approaches.

Damage caused by reactive oxygen species (ROS) is one of the potential problems when hypoxic stress occurs. Injury from prolonged hypobaric hypoxia, which is a characteristic of high altitude, is known to induce oxidative injury by increasing the generation of ROS and decreasing the effectiveness of the antioxidant defense system. However, much attention has been directed towards a 'live-high train-low' (LHTL) training approach: athletes live and sleep at high altitude, but regularly descend for training sessions to lower altitude (Levine and Stray-Gundersen 1997).

Communicated by Susan Ward.

H. Hinghofer-Szalkay $(\bowtie)$

Institute of Physiology, Centre of Physiological Medicine,

Medical University Graz, Harrachgasse 21,

8010 Graz, Austria

e-mail: helmut.hinghofer@medunigraz.at
A recent meta-analysis strikingly showed problems of heterogeneity between previous studies and calls for enhancement of protocols used, such as double-blind designs, to exclude biasing placebo, nocebo, or training-camp effects (Bonetti and Hopkins 2009).

Most hemodynamic variables, such as heart rate, cardiac output, peripheral resistance and systemic arterial pressure, increase due to chemoreceptor-mediated sympathetic activation during acute episodes of hypoxia in humans. However, experimental evidence has suggested intermittent hypoxia conditioning to be safe for prevention and treatment of hypertension (Serebrovskaya et al. 2008). The question remains under exactly which circumstances advantages of intermittent hypoxia treatment may outweigh possible long-term risks. This dilemma has been addressed at least in part by several studies, but a general consensus is still lacking. More well-controlled human investigations that focus on long-term perspectives within this framework are clearly needed. As far as only performance increase in athletes is targeted, ethical caveats linger, and should be seen as an important part of the equation.

\section{References}

Bonetti DL (2009) Hopkins WG (2009) Sea-level exercise performance following adaptation to hypoxia: a meta-analysis. Sports Med 39:107-127

Gomez-Gallego F, Santiago C, Gonzalez-Freire M, Muniesa CA, del Valle MF, Perez M, Foster C, Lucia A (2009) Endurance performance: genes or gene combinations? Int J Sports Med 30:66-72

Levine BD, Stray-Gundersen J (1997) 'Living high-training low': effect of moderate-altitude acclimatization with low-altitude training on performance. J Appl Physiol 83:102-112

Serebrovskaya TV, Manukhina EB, Smith ML, Downey HF, Mallet RT (2008) Intermittent hypoxia: cause of or therapy for systemic hypertension? Exp Biol Med 233:627-650 